\title{
Early On-Orbit Performance of the Visible Infrared Imaging Radiometer Suite Onboard the Suomi National Polar-Orbiting Partnership (S-NPP) Satellite
}

\author{
Changyong Cao, Frank J. De Luccia, Xiaoxiong Xiong, Member, IEEE, Robert Wolfe, and Fuzhong Weng
}

\begin{abstract}
The Visible Infrared Imaging Radiometer Suite (VIIRS) is one of the key environmental remote-sensing instruments onboard the Suomi National Polar-Orbiting Partnership spacecraft, which was successfully launched on October 28 , 2011 from the Vandenberg Air Force Base, California. Following a series of spacecraft and sensor activation operations, the VIIRS nadir door was opened on November 21, 2011. The first VIIRS image acquired signifies a new generation of operational moderate resolution-imaging capabilities following the legacy of the advanced very high-resolution radiometer series on NOAA satellites and Terra and Aqua Moderate-Resolution Imaging Spectroradiometer for NASA's Earth Observing system. VIIRS provides significant enhancements to the operational environmental monitoring and numerical weather forecasting, with 22 imaging and radiometric bands covering wavelengths from 0.41 to 12.5 microns, providing the sensor data records for 23 environmental data records including aerosol, cloud properties, fire, albedo, snow and ice, vegetation, sea surface temperature, ocean color, and nigh-time visible-light-related applications. Preliminary results from the on-orbit verification in the postlaunch check-out and intensive calibration and validation have shown that VIIRS is performing well and producing high-quality images. This paper provides an overview of the onorbit performance of VIIRS, the calibration/validation (cal/val) activities and methodologies used. It presents an assessment of the sensor initial on-orbit calibration and performance based on the efforts from the VIIRS-SDR team. Known anomalies, issues, and future calibration efforts, including the long-term monitoring, and intercalibration are also discussed.
\end{abstract}

Index Terms - Earth observing satellite, imaging radiometer, postlaunch calibration/validation, remote sensing, Suomi-NPP, VIIRS.

\section{INTRODUCTION}

$\mathbf{T}$ HE JOINT Polar Satellite System (JPSS) program was established in year 2010 as a result of a restructuring of the National Polar-Orbiting Environmental Satellite Sys-

Manuscript received August 14, 2012; revised January 29, 2013; accepted February 11, 2013. Date of publication May 30, 2013; date of current version December 12, 2013. This work was supported in part by the JPSS Program Office.

C. Cao and F. Weng are with NOAA/NESDIS/STAR, College Park, MD 20740 USA (e-mail: changyong.cao@noaa.gov; fuzhong.weng@noaa.gov).

F. J. De Luccia is with The Aerospace Corporation, Los Angeles, CA 90009 USA (e-mail: frank.j.deluccia@aero.org).

$\mathrm{X}$. Xiong and R. Wolfe are with the Sciences and Exploration Directorate, NASA Goddard Space Flight Center, Greenbelt, MD 20771 USA (e-mail: xiaoxiong.xiong-1@nasa.gov; robert.e.wolfe@nasa.gov).

Color versions of one or more of the figures in this paper are available online at http://ieeexplore.ieee.org.

Digital Object Identifier 10.1109/TGRS.2013.2247768 tem (NPOESS), to provide the continuity for NOAA's polarorbiting operational environmental satellite system (POES). As a result of the restructuring, the Office of Science and Technology directed NOAA and NASA to develop a mission that addresses the afternoon orbit (local Equator crossing time $\sim 1: 30$ P.M.-ascending node) data collection with a 16-day repeat cycle, while the European Organisation for the Exploitation of Meteorological Satellite (EUMETSAT) is responsible for the midmorning orbit with their MetOp series, which carry both NOAA and EUMETSAT instruments, and the Department of Defense (DoD) would be responsible for the early-morning orbit (http://npoess.noaa.gov/About/ NPOESS_Decision_Fact_Sheet_20100201.pdf).

In the early days of planning for NPOESS, it was deemed appropriate and necessary to perform a risk reduction in the development, implementation, launch, and operation of the NPOESS through its preparatory project (NPP)—renamed in early 2012 to Suomi National Polar-orbiting Partnership (S-NPP) - which provides an opportunity to demonstrate and validate new instruments and data processing algorithms, as well as to demonstrate and validate aspects of the JPSS command, control, communications, and ground-processing capabilities prior to the launch of the first JPSS spacecraft. After the launch, NASA conducted an extensive engineering evaluation and checkout of each instrument. During the instrument checkout and intensive cal/val periods, the teams led by NASA and NOAA have worked together to analyze the engineering data to verify that the key instrument-performance parameters meet the specifications. Upon completion of the testing and validation of the sensor data record (SDR), the data will be used in routine operations for the numerical weather prediction and by other user communities for 24 hours a day, 7 days a week uninterrupted. This transition is currently estimated to occur in late 2013 although the specific dates have yet to be finalized.

The Visible/Infrared Imaging Radiometer Suite (VIIRS) is one of the key earth-observing instruments onboard S-NPP and JPSS [1]-[5]. The VIIRS observations primarily focus on clouds and earth surface variables, while the other instruments, including the Cross-Track Infrared Sounder, Ozone Mapping and Profiling Suite, Advanced Technology Microwave Sounder, and Cloud and Earth Radiance Energy System (CERES) are designed to measure atmospheric variables and the earth's radiation budget. 
TABLE I

VIIRS EDRS

\begin{tabular}{|c|l|}
\hline Land -9 & Active Fires \\
& Land Surface Albedo \\
& Land Surface Temperature \\
& Ice Surface Temperature \\
& Snow Ice Characterization \\
& Snow Cover \\
& Vegetation Index \\
& Surface Type \\
& Net Heat Flux \\
\hline Ocean -2 & Sea Surface Temperature (KPP) \\
& Ocean Color/Chlorophyll \\
\hline Imagery-1 & Imagery (KPP) \\
and Clouds -7 & Cloud Optical Thickness \\
& Cloud Effective Particle Size \\
& Cloud Top Pressure \\
& Cloud Top Height \\
& Cloud Top Temperature \\
& Cloud Base Height \\
& Cloud Cover/Layers \\
\hline Aerosols -3 & Aerosol Optical Thickness \\
& Aerosol Particle Size \\
& Suspended Matter \\
\hline Low Light Imaging -1 & Near Constant Contrast (NCC) Imagery \\
& \\
\hline
\end{tabular}

\section{A. VIIRS Applications and EDRs}

The VIIRS specifications and implementations require the instrument to produce data for many biogeophysical parameters, known as environmental data records (EDRs) [6]-[8]. Among the 23 EDRs, the sea surface temperature (SST) and imagery are the two key performance parameters. The EDRs fall into three broad categories of land, atmosphere, and ocean, as listed in Table I. Although there are similarities between the VIIRS-EDRs and MODIS level-2 products, VIIRS does not have any atmospheric sounding bands and related products. Nevertheless, the operational use of VIIRS signifies a major step succeeding and advancing the advanced very high-resolution radiometer (AVHRR) with more spectral bands and products and at higher spatial resolutions. Further discussion of the EDRs, their performance requirements, and their generation are beyond the scope of this paper. Data users are referred to the operational algorithm description in the document section at http://www.star.nesdis.noaa.gov/jpss/, in addition to a number of journal publications and books [6]-[10], the list for which is expected to grow in the next few years.

\section{B. VIIRS Heritage}

VIIRS was developed based on a long heritage of legacy operational and research instruments, dating back as early as the late 1970s. Key heritage instruments include: the AVHRR on NOAA's POES, the Moderate-Resolution Imaging Spectroradiometer (MODIS) on NASA's Earth-Observing System (EOS) Terra and Aqua satellites, the Sea-Viewing Wide Fieldof-View Sensor (SeaWiFS) on SeaStar, and the Operational Linescan System (OLS) on DoD's Defense Meteorological Satellite Program (DMSP).

The first AVHRR was launched on TIROS-N in 1978 with four bands, and the later models of AVHRR/3 have six bands with three in the visible/near-infrared (VNIR), and three in the infrared. The early AVHRRs observed the earth in early morning ( $\sim$ 7:30 A.M.) and afternoon ( $\sim 1: 30$ A.M.) orbits, while the early-morning orbit was changed to midmorning starting with NOAA-17 in 2002, followed by the MetOp series. The NOAA satellite orbits are not precisely controlled, which led to orbital drift and related issues over the mission life. With precision control of the S-NPP orbit, VIIRS significantly outperforms AVHRR in all aspects of spatial, spectral, and radiometric resolution and accuracy.

The MODIS is a key instrument with 36 spectral bands aboard the midmorning Terra and afternoon Aqua satellites launched in 1999 and 2002, respectively. The nadir spatial resolution of its reflective solar bands (RSBs) is as high as $250 \mathrm{~m}$, while the $1-\mathrm{km}$ nadir resolution is achieved for all the thermal emissive bands (TEBs). The MODIS onboard calibration devices for the RSB significantly improved the accuracy of the measurements, which in turn enabled a number of quantitative products. The VIIRS design was largely built upon the success of MODIS with many similar features. Major differences are that VIIRS does not have atmospheric sounding bands in comparison to MODIS, which includes fourcloud top-height bands (13-14 $\mu \mathrm{m})$, five water vapor bands (0.89-0.96 and 6.5-7.4 $\mu \mathrm{m})$, one ozone band $(9.5-9.8 \mu \mathrm{m})$, two atmospheric temperature bands $(4.4-4.5 \mu \mathrm{m})$, and two ocean color (OC) bands $(0.52$ and $0.68 \mu \mathrm{m})$ (Table II). The VIIRS dual gain design for seven bands not only reduced redundancy that exists in MODIS (such as the $0.55 \mu \mathrm{m}$ band), but also extended the dynamic range of the observations, which is especially important for the fire versus SST, and cloud versus OC applications. Two of the VIIRS-TEB-imaging bands have a much higher nadir spatial resolution $(375 \mathrm{~m}$ compared to the corresponding MODIS 1-km TEBs). Fig. 1 shows that man-made features such as harbors and canals can be observed by the TEB-imaging bands clearly at night. In addition, VIIRS also has a day-night band (DNB).

The SeaWiFS is a follow-on experiment to the coastal zone color scanner on Nimbus 7. It began scientific operations in 1997 and stopped collecting data by late 2010. Its nadir spatial resolution is $1.1 \mathrm{~km}$ with 8 spectral bands from 0.402 to $0.885 \mu \mathrm{m}$. It was specifically designed to monitor ocean characteristics such as chlorophyll-a concentration and water clarity. The SeaWiFS instrument uses a rotating telescope and a half-angle mirror, which became the heritage design of VIIRS. The VIIRS M1 to M7 bands are designed to match those of the SeaWiFS bands.

The OLS on DMSP is the operational visible/infrared scanner for the DoD. It has a low-light panchromatic sensor in the VNIR $(0.59-0.91 \mu \mathrm{m})$, and an infrared sensor covering the 10.0-13.4 $\mu \mathrm{m}$. The OLS scans across the ground with a nadir resolution of $2.7 \mathrm{~km}$, capable of detecting visible lights at night. OLS has primarily served as a data source for the manual analysis of imagery. The VIIRS-DNB has outperformed OLS in radiometric accuracy, partly due to the onboard calibration using the solar diffuser (SD), and the DNB 750-m spatial resolution surpassed that of the OLS, which allows observations of the city lights, moon-illuminated earth, and other night phenomena with much finer details (Fig. 1). 


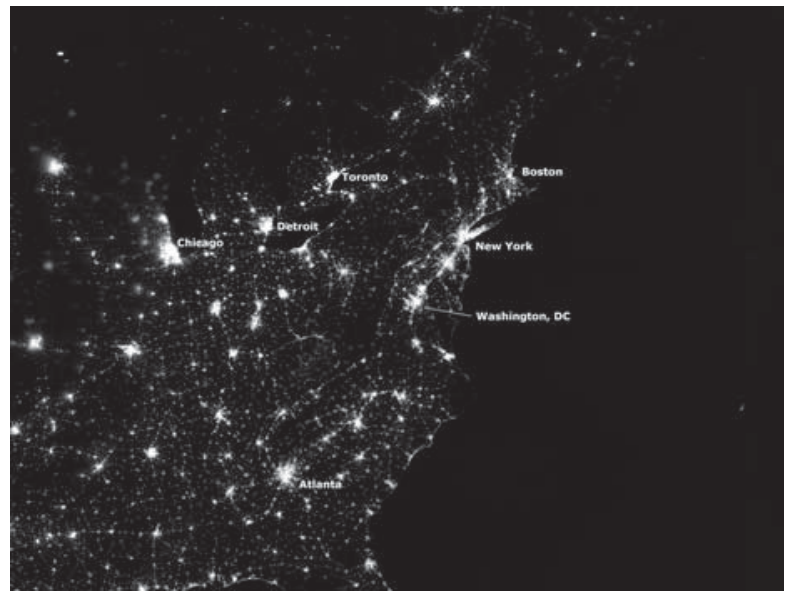

(a)

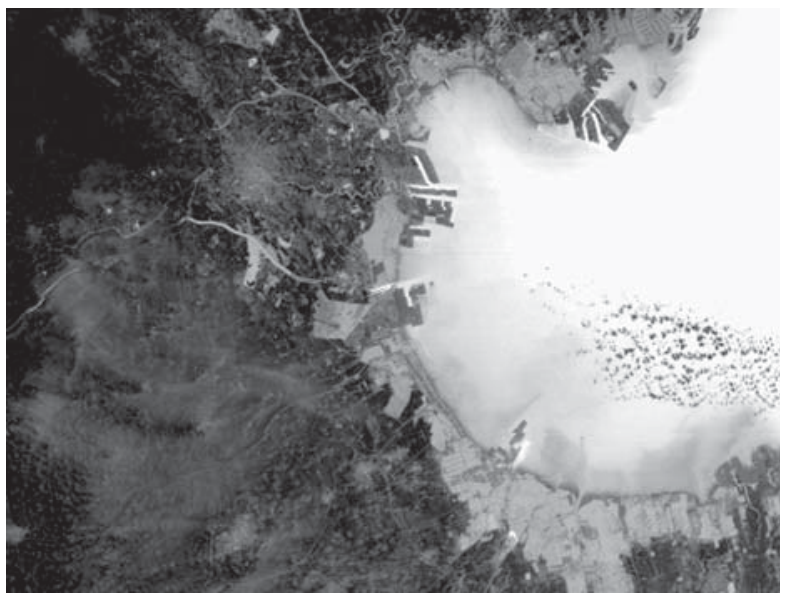

(b)

Fig. 1. VIIRS advanced night imaging capabilities. (a) DNB image of Northeast U.S. w/750 m resolution (March 27, 2012). (b) I-5 image of Tianjin harbor w/375 m resolution (11/08/2012).



Fig. 2. VIIRS instrument block diagram (after Gleason: http://modis.gsfc.nasa.gov/sci_team/meetings/201105/presentations/plenary/gleason.pptx).

\section{VIIRS ChARACTERISTICS, PRELAUNCH, AND Postlaunch Performance}

VIIRS is designed to provide moderate-resolution, radiometrically accurate images of the globe once per day for the RSBs and twice daily for the TEBs and DNB [1], [2], [12], [13]. It is a wide-swath $(3000 \mathrm{~km})$ scanning radiometer with spatial resolutions of 375 and $750 \mathrm{~m}$ at nadir for the imaging bands (aka I-bands) and moderate resolution bands (aka M-bands), respectively. The 22 spectral bands include 14 RSB, 7 TEB, and 1 DNB. The M-bands have better signal-to-noise ratio (SNR) and radiometric accuracy and are better suited for quantitative applications, while the I-bands have high spatial resolution with broader spectral response (Tables II and III).

Fig. 2 shows the block diagram of the VIIRS sensor. A brief discussion is provided here as an overview of the instrument design. Reflected and emitted radiations from the earth enter the sensor through the rotating telescope assembly (RTA) and

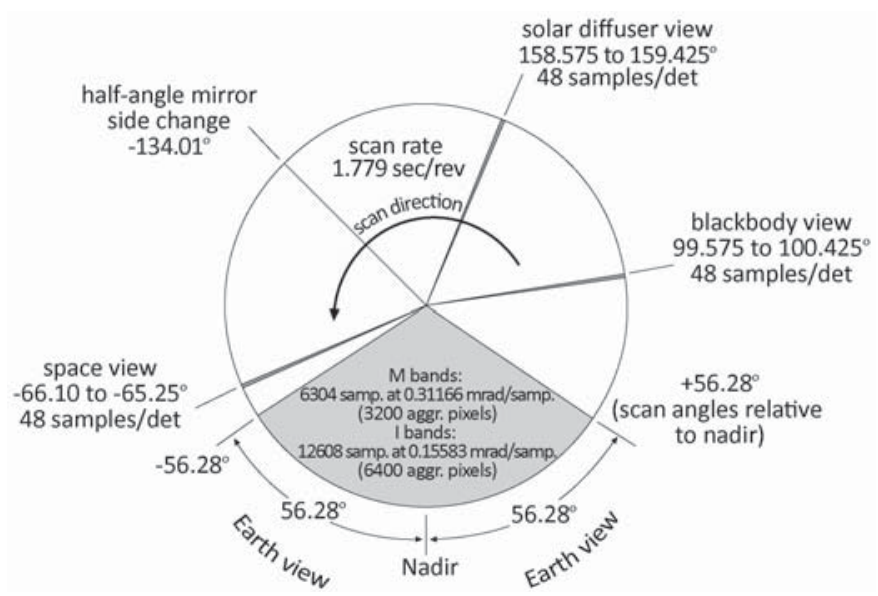

Fig. 3. VIIRS scan pattern (as built values, updated 7/2012).

is reflected from a rotating half-angle mirror (HAM) into a stationary aft-optics subsystem. The light is then spectrally and spatially separated by dichroic beamsplitters and directed 
TABLE II

VIIRS AND MODIS BAND COMPARISONS

\begin{tabular}{|c|c|c|c|c|c|c|c|c|c|}
\hline \multicolumn{5}{|c|}{ MODIS } & \multicolumn{5}{|c|}{ VIIRS } \\
\hline Band & $\lambda(\mu \mathrm{m})$ & Ltyp/Ttyp & \begin{tabular}{|c|} 
SPEC \\
SNR/NEdT/ \\
$($ NEdL)
\end{tabular} & $\begin{array}{c}\text { On Orbit } \\
\text { SNR/NEdT/ } \\
\text { (NEdL) }\end{array}$ & Band & $\lambda(\mu \mathrm{m})$ & Ltyp & $\begin{array}{c}\text { SPEC } \\
\text { SNR/NEdT } \\
/(\text { NEdL) }\end{array}$ & $\begin{array}{l}\text { On Orbit } \\
\text { SNR/NEdT } \\
\text { /(NEdL) }\end{array}$ \\
\hline 1 & $0.620-0.670$ & 21.8 & 128 & 198.40 & $\mathrm{I}-1$ & $0.597-0.679$ & 22 & 119 & 214 \\
\hline 2 & $0.841-0.876$ & 24.7 & 201 & 510.54 & $\mathrm{I}-2$ & $0.842-0.881$ & 25 & 150 & 264 \\
\hline 3 & $0.459-0.479$ & 35.3 & 243 & 320.76 & & & & & \\
\hline 4 & $0.545-0.565$ & 29.0 & 228 & 323.76 & M-4 & $0.541-0.561$ & 90 & 315 & 856 \\
\hline 5 & $1.230-1.250$ & 5.4 & 74 & 151.70 & M-8 & $1.225-1.252$ & 5.4 & 74 & 221 \\
\hline \multirow[t]{2}{*}{6} & \multirow[t]{2}{*}{$1.628-1.652$} & \multirow[t]{2}{*}{7.3} & \multirow[t]{2}{*}{275} & \multirow[t]{2}{*}{456.50} & M-10 & $1.571-1.631$ & 7.3 & 342 & 586 \\
\hline & & & & & $\mathrm{I}-3$ & $1.570-1.629$ & 7.3 & 6 & 149 \\
\hline 7 & $2.105-2.155$ & 1.0 & 110 & 154.00 & M-11 & $2.234-2.280$ & 0.12 & 10 & 22 \\
\hline 8 & $0.405-0.420$ & 44.9 & 880 & 1117.60 & M-1 & $0.400-0.421$ & 44.9 & 352 & 588 \\
\hline 9 & $0.438-0.448$ & 41.9 & 838 & 1541.92 & M-2 & $0.436-0.451$ & 40 & 380 & 572 \\
\hline 10 & $0.483-0.493$ & 32.1 & 802 & 1563.90 & M-3 & $0.477-0.496$ & 32 & 416 & 628 \\
\hline 11 & $0.526-0.536$ & 27.9 & 754 & 1726.66 & & & & & \\
\hline 12 & $0.546-0.556$ & 21.0 & 750 & 1522.50 & & & & & \\
\hline 13 & $0.662-0.672$ & 9.5 & 910 & 1437.80 & M-5 & $0.662-0.680$ & 10 & 242 & 336 \\
\hline 14 & $0.673-0.683$ & 8.7 & 1087 & 1576.15 & & & & & \\
\hline 15 & $0.743-0.753$ & 10.2 & 586 & 1570.48 & M-6 & $0.738-0.752$ & 9.6 & 199 & 368 \\
\hline 16 & $0.862-0.877$ & 6.2 & 516 & 1439.64 & M-7 & $0.843-0.881$ & 6.4 & 215 & 457 \\
\hline 17 & $0.890-0.920$ & 10.0 & 167 & 367.40 & & & & & \\
\hline 18 & $0.931-0.941$ & 3.6 & 57 & 91.20 & & & & & \\
\hline 19 & $0.915-0.965$ & 15.0 & 250 & 507.50 & & & & & \\
\hline \multirow[t]{2}{*}{20} & \multirow[t]{2}{*}{$3.660-3.880$} & \multirow[t]{2}{*}{300} & \multirow[t]{2}{*}{$0.05(0.0010)$} & \multirow[t]{2}{*}{$0.024(0.0005)$} & M-12 & $3.598-3.791$ & 270 & $0.396(0.002)$ & $0.12(0.00059)$ \\
\hline & & & & & $\mathrm{I}-4$ & $3.550-3.937$ & 270 & $2.5(0.014)$ & $0.4(0.0023)$ \\
\hline 21 & $3.929-3.989$ & 335 & $0.20(0.0150)$ & $0.183(0.0145)$ & & & & & \\
\hline 22 & $3.940-4.001$ & 300 & $0.07(0.0019)$ & $0.019(0.0005)$ & & & & & \\
\hline 23 & $4.020-4.080$ & 300 & $0.07(0.0022)$ & $0.020(0.0006)$ & M-13 & $3.987-4.145$ & 300 & $0.107(0.0033)$ & $0.04(0.00128)$ \\
\hline 24 & $4.433-4.498$ & 250 & $0.25(0.0022)$ & $0.109(0.0009)$ & & & & & \\
\hline 25 & $4.482-4.549$ & 275 & $0.25(0.0062)$ & $0.039(0.0010)$ & & & & & \\
\hline 26 & $1.360-1.390$ & 6.0 & 150 & 277.50 & M-9 & $1.368-1.383$ & 6 & 83 & 227 \\
\hline 27 & $6.535-6.895$ & 240 & $0.25(0.0108)$ & $0.085(0.0038)$ & & & & & \\
\hline 28 & $7.175-7.475$ & 250 & $0.25(0.0172)$ & $0.041(0.0029)$ & & & & & \\
\hline 29 & $8.400-8.700$ & 300 & $0.05(0.0090)$ & $0.019(0.0034)$ & M-14 & $8.407-8.748$ & 270 & $0.091(0.011)$ & $0.06(0.00651)$ \\
\hline 30 & $9.580-9.880$ & 250 & $0.25(0.0219)$ & $0.089(0.0078)$ & & & & & \\
\hline \multirow[t]{2}{*}{31} & \multirow[t]{2}{*}{$10.780-11.280$} & \multirow[t]{2}{*}{300} & \multirow[t]{2}{*}{$0.05(0.0070)$} & \multirow[t]{2}{*}{$0.017(0.0023)$} & M-15 & $10.234-11.248$ & 300 & $0.07(0.01)$ & $0.03(0.00392)$ \\
\hline & & & & & $\mathrm{I}-5$ & $10.560-12.428$ & 210 & $1.5(0.066)$ & $0.4(0.0171)$ \\
\hline 32 & $11.770-12.270$ & 300 & $0.05(0.0061)$ & $0.025(0.0031)$ & M16 & $11.405-12.322$ & 300 & $0.072(0.0087)$ & $0.03(0.00355)$ \\
\hline 33 & $13.185-13.485$ & 260 & $0.25(0.0183)$ & $0.082(0.0060)$ & & & & & \\
\hline 34 & $13.485-13.785$ & 250 & $0.25(0.0161)$ & $0.122(0.0078)$ & & & & & \\
\hline 35 & $13.785-14.085$ & 240 & $0.25(0.0141)$ & $0.156(0.0088)$ & & & & & \\
\hline 36 & $14.085-14.385$ & 220 & $0.35(0.0154)$ & $0.230(0.0101)$ & & & & & \\
\hline
\end{tabular}

Note:

-Unit for Ltyp and NEdL is $\mathrm{W} /\left(\mathrm{m}^{2}-\mathrm{sr}-\mu \mathrm{m}\right)$; Unit for Ttyp and NEdT is $\mathrm{K}$.

-For TEB, both NEdT and NEdL (in parenthesis) are provided.

-VIIRS spectral range values are as built for S-NPP/VIIRS.

-VIIRS Bands I1-I5 resolution is $375 \mathrm{~m}$ at Nadir; Bands M1-M16 resolution is $750 \mathrm{~m}$ at Nadir.

-MODIS Bands 1-2 are $250 \mathrm{~m}$ at Nadir; Bands 3-7 are $500 \mathrm{~m}$ at Nadir; Bands 8-36 are $1000 \mathrm{~m}$ at Nadir.

to three separate focal plane arrays (FPAs): the VNIR-FPA, the shortwave/midwave infrared (SW/MWIR)-FPA, and the longwave infrared (LWIR)-FPA. The light is detected and converted to analog electrical signals in these FPAs and further processed prior to analog-to-digital (A/D) conversion with a 14-bit quantization, truncated to 12 bits for the earth scan data. The digital signals are then processed and multiplexed into the instrument-output data stream. Housekeeping data in the form of instrument health, safety, and engineering telemetry are also generated from measurements of internal tempera- tures, voltages, and currents. These telemetry measurements are reported for each scan and downlinked to the groundprocessing system.

The VIIRS scan pattern is presented in Fig. 3. It scans the earth, blackbody, SD, and space view (SV) in sequence. The HAM changes side at $-134.01^{\circ}$ between the SD and SV. The earth-view scan angle range is $\pm 56.28^{\circ}$ from nadir, which provides a full coverage of $3000 \mathrm{~km}$ with no gap between orbits - an advantage over MODIS (with 2300-km swath width) and other instruments. VIIRS is calibrated every 
TABLE III

VIIRS SPECTRAL, SPATIAL, AND RADIOMETRIC CHARACTERISTICS

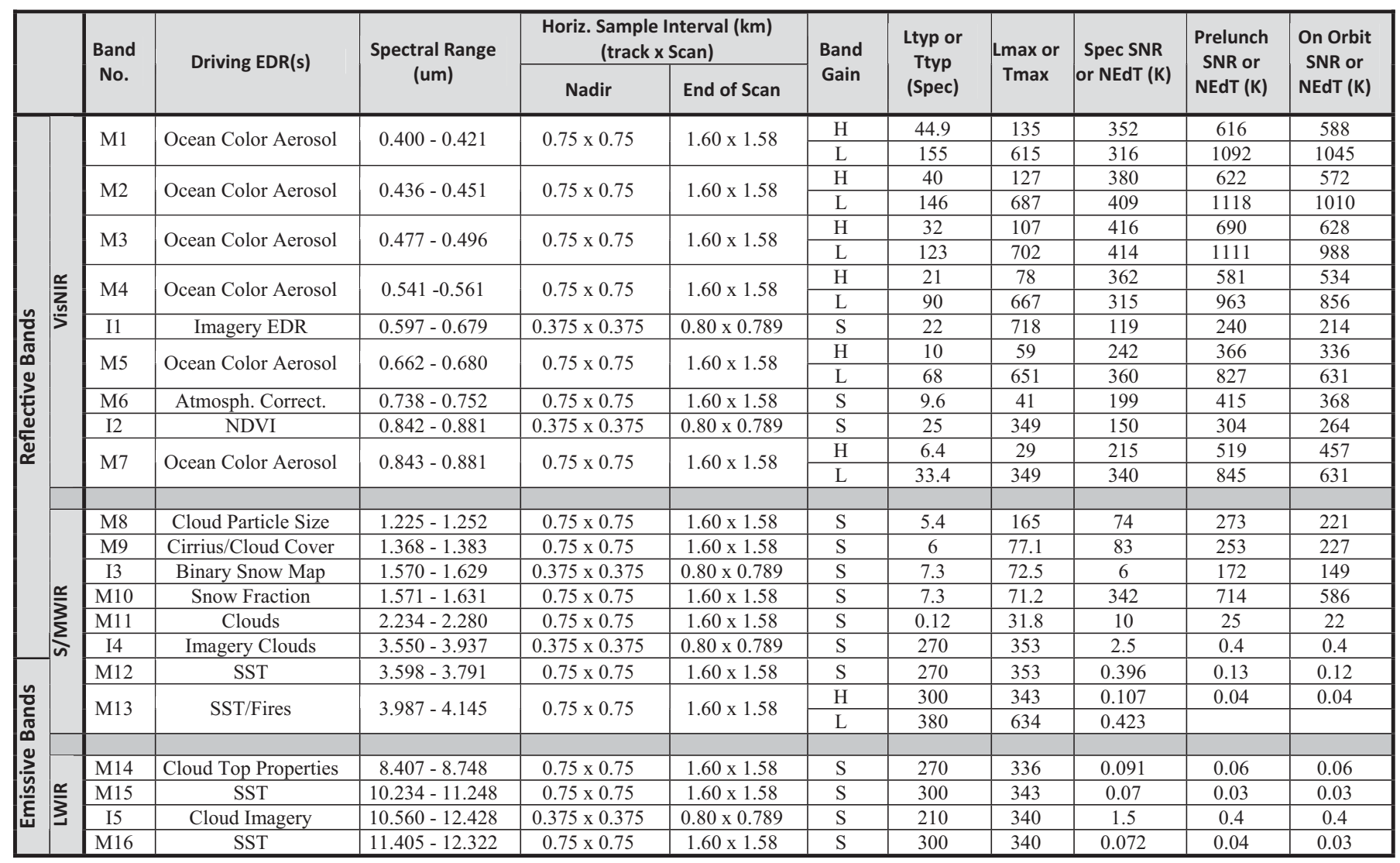

Note:

On-orbit values are as of April 5, 2012; Ltyp unit: W/m ${ }^{2}$-sr- $\mu \mathrm{m}$, Ttyp unit: K; Band Gain: $\mathrm{H}=\mathrm{High}, \mathrm{L}=\mathrm{Low}, \mathrm{S}=$ Single.

The horizontal sample interval (HSI) values shown are nominal values. The actual values vary with specific spacecraft altitude and location.

scan-line for the TEB, while the RSB calibration relies on the solar illumination in part of every orbit. Calibration lookup tables (LUTs), which are based on prelaunch measurements updated with these on-orbit measurements, are used in the ground-processing system to produce calibrated VIIRS-SDR. All of the calibration-related data are stored in the onboard calibrator intermediate product (OBC IP) files, which are used for the long-term monitoring (LTM) of the instrument performance.

As noted earlier, the heritage of the RTA design of VIIRS came from the SeaWiFS, which provides better straylight control at high scan angles, while at the same time reducing the response versus scan angle effects (MODIS paddle mirror versus VIIRS HAM). The higher S-NPP and JPSS orbits (equatorial altitude $829 \mathrm{~km}$ versus $705 \mathrm{~km}$ for the EOS terra and aqua platforms) allow full global coverage twice daily but also require better stray-light controls.

\section{A. VIIRS Spectral Bands}

The VIIRS spectral bands are located on three focal planes. There are 16 detectors for each M-band and 32 detectors for each I-band, aligned in the along-track direction. Therefore, each scan acquires 16 or 32 scan-lines (similar to MODIS but in contrast to the one-per-scan-line pattern of AVHRR), which increases the integration time to achieve stronger signals. The RSB focal plane is operated at instrument ambient temperature $(\sim 270 \mathrm{~K})$, while the short/mid wave infrared and TEB-FPAs are cooled down to $80 \mathrm{~K}$ with a passive radiative cooler.

For the RSB, VIIRS uses six dual-gain bands to provide the high radiometric resolution needed for OC applications, while not saturating the sensor when observing high-reflectance surfaces from the land and clouds. The dynamic range of the dual-gain bands in high gain is comparable to that of the MODIS OC bands, while the dynamic range in the low-gain state is comparable to those of the similar MODIS land bands. The dynamic ranges across all other bands are similar to their MODIS counterparts. Among the TEB, dual gain is used for M13 to meet the needs of both SST monitoring and fire detection at the same time (Tables II and III). By design, transitions from high gain (low radiance such as over ocean) to low gain (high radiance such as over land and cloud) are performed automatically at the focal plane electronics level based on a switch-point bias voltage that is supplied by the analog signal processor, which is part of the electronics module (https://cs.star.nesdis.noaa.gov/NCC/VIIRSCalATBD). 


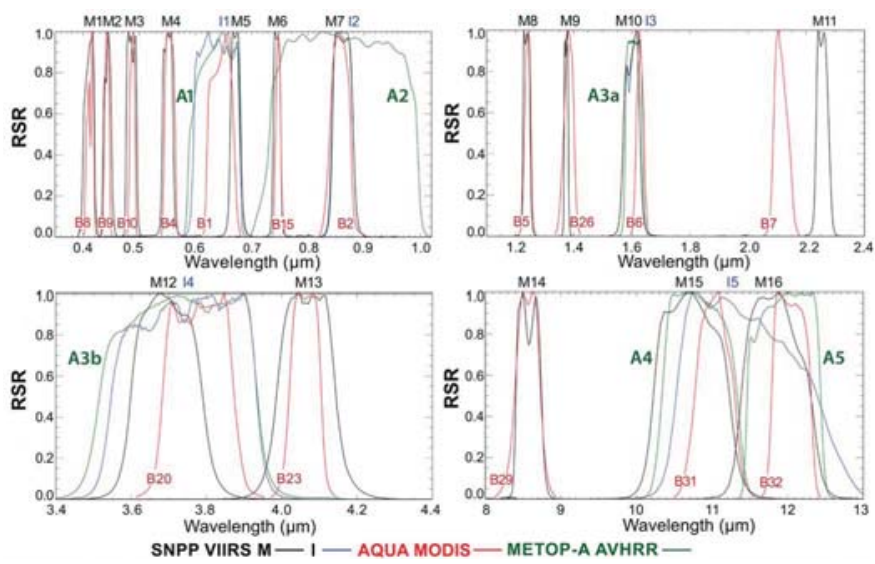

Fig. 4. VIIRS relative spectral response functions in comparison with that of MODIS and AVHRR.

The VIIRS panchromatic DNB-band measures the surfacereflected solar- and/or moon-visible radiation with a large dynamic range of $45000000: 1$, which enables the detection of reflected signals from as low as quarter moon illumination to the brightest daylight [4], [11]. To achieve this large dynamic range, it uses a three-stage focal plane. The sensor maintains a nearly constant $750-\mathrm{m}$ resolution over the entire $3000-\mathrm{km}$ swath using an on-board aggregation scheme. On the other hand, this scheme also makes the calibration of the DNB band a challenge.

The VIIRS spectral response is shown in Fig. 4 overlaid with that of AVHRR and MODIS for comparison. Major efforts have been devoted to the characterization of the S-NPP-VIIRS spectral response functions (or relative spectral response, aka, RSR) for the RSB [14], [15]. They are first measured at the instrument level during laboratory testing in the thermal vacuum chamber. Then they are measured at spacecraft level with the state-of-the-art technology of the spectral irradiance and radiance responsivity calibrations using uniform sources (SIRCUS) from the National Institute of Standards and Technology (NIST). The measurement results have been rigorously compared, discrepancies analyzed and resolved, and the impact on products evaluated. The final RSR in digital files are now available at https://cs.star.nesdis.noaa.gov/NCC/VIIRS.

As can be seen from Fig. 4, in general, the VIIRS I-bands have broader spectral response than those of the M-bands. The band center wavelengths are comparable to those of MODIS and SeaWiFS. When compared to MODIS qualitatively, most of the VIIRS-bands have wider band width for the RSB. The VIIRS near-infrared band spectral responses are comparable to those of MODIS, except for the $1.38-\mu \mathrm{m}$ band, which is much narrower. The TEBs at 4.05, 10.8, and $12.0 \mu \mathrm{m}$ are doubled in bandwidth to that of MODIS, while the $8.55-\mu \mathrm{m}$ band is similar. The broader bandwidth increases the throughput of the photons reaching the detectors, which are needed for the higher spatial resolution while maintaining the SNR or NEdT.

As shown in Table III, the M1-M7 bands are used primarily for OC and aerosol applications: the M8, M9, M11, M14, I4, and I5 bands for clouds; M12, M13, M15 and M16 primarily for SST (M13 also for fire); I3 and M10 for snow; I1 and I2 for normalized difference vegetation index (NDVI) and imagery.
The typical radiance (Ltyp) or brightness temperature (Ttyp) are provided in Tables II and III for reference. It should be noted that when comparing the SNR values between VIIRS and MODIS, the Ltyp and Ttyp values at which the SNR and NEdT are specified should be considered since they may have different Ltyp or Ttyp values. For example, in Table II, the MODIS SNR of 323.76 for B4 is at Ltyp of $29 \mathrm{~W} /$ $\left(\mathrm{m}^{2}-\mathrm{sr}-\mu \mathrm{m}\right)$, while the VIIRS SNR of 856 for M4 is at Ltyp of $90 \mathrm{~W} /\left(\mathrm{m}^{2}-\mathrm{sr}-\mu \mathrm{m}\right)$.

\section{B. VIIRS Early On-Orbit Radiometric Performance}

The postlaunch calibration/validation process undergoes three phases [16]: Early orbit checkout (EOC), intensive calibration/validation (ICV), and LTM. Typically, EOC is the first 3-6 months after the instrument is turned on. During this period, data will be available only to team members such as the SDR and EDR teams. This "beta" maturity period features early release products with initial calibration applied, and minimally validated, but may still contain significant errors and more frequent changes can be expected. Nevertheless, products are available to allow users to gain familiarity with data formats and parameters for evaluation. Products may not be appropriate for the evaluation against requirements or quantitative scientific research and applications.

ICV is the period from the end of EOC to plus approximately 2-6 months. During this "provisional" maturity period, the SDR data are evaluated for operational use. "Provisional" means that product quality may not be optimal. Incremental product improvements are still occurring as calibration parameters are adjusted with the sensor on-orbit characterization. The research community is encouraged to participate in the quality assessment and validation of the products, but need to be aware that these activities are ongoing. Toward the end of this provisional period, the data product is considered validated. The official transition to the operations phase is expected to begin at launch plus 9 to 15 months.

LTM is the period from the end of ICV until the end of the mission when the data are mature for operational use, although anomalies and upgrades are still expected. Also, the data may be reprocessed when the SDRs and EDRs can be improved significantly as they progress through the stages of validation described above as an iterative process. However, reprocessing is not in the current baseline of the S-NPP/JPSS program and will require additional efforts and resources.

The S-NPP was launched on October 28, 2011. Following a series of spacecraft and sensor activation and checkouts, the first VIIRS image was acquired on November 21, 2011, and all 22 VIIRS bands were producing early images by January 20, 2012. Since launch, the VIIRS-SDR calibration/validation has been progressing well [17]-[20]. A team of experts from NOAA, NASA, the Aerospace Corporation (Aerospace), University of Wisconsin, MIT/Lincoln Laboratory, and other industry partners have worked intensively and performed a thorough evaluation of the VIIRS on-orbit performance with $58 \mathrm{cal} / \mathrm{val}$ tasks [14], [15]. These tasks include seven tasks for the functional performance and format evaluation, seven tasks for calibration system evaluation, four tasks for image quality evaluation, 25 tasks for radiometric evaluation, nine tasks 
for geometric evaluation, and five tasks for performance and telemetry trending. Descriptions of these tasks can be found in the VIIRS SDR operational concept (OPSCON) document [16].

On April 5, 2012, a VIIRS-SDR review meeting was held. After a thorough review, the VIIRS-SDR team, EDR users, and the review panel members reached consensus that the VIIRS-SDR product overall had reached beta status and therefore was recommended to be made available to the public through the Comprehensive Large Array-Data Stewardship System (CLASS). Release of pre-beta VIIRS-SDR data are expected to remain restricted to the SDR and EDR teams. As of mid-December 2012, the VIIRS-SDR is achieving provisional status, pending official approval and public announcement. The following sections provide a summary of the instrument performance evaluation since launch.

1) VIIRS Onboard Calibration System: To meet the radiometric performance requirements through the entire mission, the onboard calibration devices are essential for VIIRS. The calibration source for RSB is a full-aperture SD that is illuminated once per orbit as the satellite passes from the dark side to the sunlit side of the earth in the high latitudes of the southern hemisphere. An attenuation screen covers the opening, but there is no door or other optical element between the SD and the sun. The bidirectional reflectance distribution function (BRDF) of the SD and the transmittance of the attenuation screen are measured prelaunch and verified onorbit through observations made during spacecraft maneuvers. Given the angles of incidence, the reflected solar radiance can be computed and is used as a reference to produce calibrated reflectance and radiance. The SV provides the offset measurements needed for the calibration. Based on the postlaunch calibration/validation, the VIIRS-SD is stable over most of the visible and near-infrared spectrum but shows a moderate degradation toward the blue spectral region. For example, a $\sim 10 \%$ SD degradation has been observed for the first six months in the $0.412-\mu \mathrm{m}$ spectral region, primarily due to ultraviolet radiation on the $\mathrm{SD}$, while little degradation is observed in the longer wavelength such as the $0.865-\mu \mathrm{m}$ spectral region. This VIIRS-SD degradation is more pronounced than that of Aqua MODIS, which is $\sim 2.6 \%$ per year at $0.412 \mu \mathrm{m}$ [21]-[23] mainly due to the more frequent SD exposure to the sun light since there is no SD door. The SD degradation is monitored by the SD stability monitor (SDSM), which is a separate device with eight detectors (from 0.412 to $0.926 \mu \mathrm{m})$. It uses the sun as the reference and views the sun and the SD to determine the degradation of the SD, which in turn is used to compensate for the SD degradation in the onboard calibration. In the postlaunch evaluation, it was found that these detectors in the SDSM themselves also degrade over time, although their impact on the SD monitoring is effectively canceled out in the algorithm that estimates SD degradation. The SDSM spectral degradation pattern shows that unlike the SD degradation, the SDSM detectors degrade more in the longer wavelength than in the shorter wavelength. For example, detector 8 at $0.926 \mu \mathrm{m}$ degraded $>15 \%$ in the first six months, while the detector at $0.555 \mu \mathrm{m}$ had no noticeable degradation during the same time-period.
The faster degradation in the longer wavelength is likely due to preferential bombardment by high-energy particles that affect more in the longer wavelength than in the shorter wavelength because these particles can penetrate deeper in the detector layers of the FPA [24]. This mechanism is similar to that of the hypothesized SeaWiFS band degradation over time (Gene Eplee, 2011, personal communications). The technical details of the SD and SDSM are beyond the scope of this paper, but since they are very similar in both design and performance to the SD and SDSM on MODIS, related references can be found in several publications [21]-[23], [25], [26].

For the RSB, the calibration uncertainty in spectral reflectance for a scene at typical radiance is expected to be less than $2 \%$ [12], [13]. This performance has been demonstrated in the prelaunch testing in the laboratory, but the on-orbit performance requires additional effort by using the onboard SD, and vicarious calibration at desert and ocean sites, as well as intercomparisons with other satellite instruments. Additionally, the monthly lunar calibration through a spacecraft roll maneuver is part of the postlaunch calibration strategy to ensure that the sensor degradation is independently verified [27].

The TEBs are calibrated with an onboard calibrator blackbody (OBC BB) that resembles that of the MODIS [35] and has been carefully characterized at the prelaunch. The BB temperature is controlled using heater elements and thermistors. The calibration algorithm, based on the measured BB temperature, emissivity, and spaceview, computes the blackbody radiances and relates them to counts to determine the gain [28]-[30]. Variations in background emission from the halfangle mirror, and components in the surround, are taken into account in scan-angle dependent corrections, as shown in the calibration equations [29].

The TEB calibration can be affected by the thermal dynamics of the VIIRS instrument components and surround. In general, the VIIRS instrument component temperatures are very stable. The largest daily temperature variation is observed for the telescope bulkhead, which oscillates between $264 \mathrm{~K}$ and $268 \mathrm{~K}$, every orbit. Other components in the fore-optics have a temperature orbital variation of the order of $0.1 \mathrm{~K}$. Two of the platinum resistance thermometers (PRT) on the OBC $\mathrm{BB}$ have an orbital temperature variation of $0.03 \mathrm{~K}$ with a peak during the day time. This behavior is believed to reflect nonuniform variations in the effective temperature of the $\mathrm{OBC}$ BB-radiating surface. Since the current operational algorithm treats all six PRT values equally, it does not compensate for temporally varying gradients in the $\mathrm{OBC} B \mathrm{~B}$ effective temperature. The impact of these effects on the data products such as SST is estimated to be below $0.03 \mathrm{~K}$ and will be further investigated. The VIIRS long-wave cold focal plane temperature is very stable at $79.94 \mathrm{~K}$ with no noticeable variations over time. Detailed LTM of VIIRS telemetry is accessible online from https://cs.star.nesdis.noaa.gov/NCC/VIIRS.

2) VIIRS Instrument Noise Characterization: The prelaunch and on-orbit performance of the 21 VIIRS bands are summarized in Table III, which shows that the SNR or the noiseequivalent change in temperature (NEdT) for all VIIRS bands far exceeded the requirements and specifications. For the 

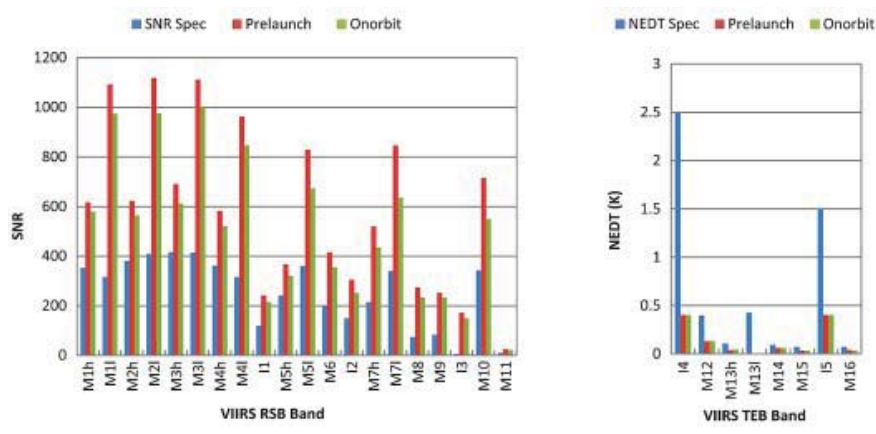

Fig. 5. VIIRS noise performance comparison: specification, prelaunch, and on-orbit.

RSB, the prelaunch SNR was derived from the VIIRS system response to the Spherical Integrator Source-100 calibrator at the radiance level corresponding to Ltyp. The Ltyp values for each band are also presented in Table III. Prior to launch, the TEB NEdT was derived from the VIIRS instrument response to the laboratory blackbody calibration source (BCS) radiance at Ltyp, which is traceable to NIST radiance standards through the temperature scale of the BCS-PRTs, using a modeled emissivity (greater than 0.9999), and assuming equivalence of temperature and radiance scales to within the required uncertainty. The BCS was operated over the temperature range of 190-340 K, during which the VIIRS instrument response was recorded to evaluate instrument performance with temperature to determine the effects such as nonlinearity.

Postlaunch results from the on-orbit calibration data show that VIIRS noise performance is excellent, exceeding specifications for all bands (Tables II and III) [18]-[20]. SNRs at typical scene radiance for the reflected solar bands (or RSB M1-M11, I1-I3, and DNB) are all more than $30 \%$ better than the specification, and several times better than specification for selected bands. For the RSB on-orbit performance, the SNR was derived from SD view and SV observations. The noise equivalent delta radiance (NEdL) are computed using the SD and SV data as the standard deviation of the signal measured in digital number (DN) for the constant radiance scenes of either the SD or SV views. The NEdL and thus the SNR at Ltyp were then interpolated (extrapolated) from the NEdL at these known levels. However, it should be noted that as discussed in the next section, the SNR for bands affected by the RTA optical throughput degradation (such as M7) are decreasing over time.

The postlaunch noise equivalent delta temperature (NEdT) for the TEB (M12-M16, and I4-I5) is also more than $30 \%$ better than specifications. This is consistent with prelaunch test results as shown in Fig. 5. For the on-orbit performance, TEB-NEdT is determined from the VIIRS instrument response to the $\mathrm{OBC} \mathrm{BB}$, which operates at a nominal temperature of $\sim 292 \mathrm{~K}$. The NEdL is computed at the blackbody temperature, interpolated to the specified Ltyp level, and then the NEdL is converted to its corresponding NEdT at Ttyp.

It should be noted that the NEdL values at blackbody and SVs are comparable for the TEB. In other words, the noise is not a significant function of the amount of incoming radiation. Therefore, the NEdT at different scene brightness temperatures



Fig. 6. VIIRS TEB band noise versus scene brightness temperature. Note: NEdL (W/m2-sr-m) for: M12 $=0.00059 ; \mathrm{M} 13=0.00128 ; \mathrm{M} 14=0.00651$; $\mathrm{M} 15=0.00392 ; \mathrm{M} 16=0.00355 ; \mathrm{I} 4=0.0023 ; \mathrm{I} 5=0.0171)$.

can be calculated using the Planck function. Fig. 6 provides the NEdT at different brightness temperatures. As discussed later, all performance values presented here are for VIIRS B-side electronics, which was better-characterized prelaunch than the A-side.

Striping between scans is a common concern for radiometers with detector's arrays due to the detector-to-detector differences. A number of destriping studies have been performed for the predecessor MODIS RSB and TEB due to the well-publicized MODIS-striping issues. Before the launch of S-NPP-VIIRS, striping was one of the major concerns partly due to the nonuniform sensitivity across detectors to polarized signals toward the blue bands measured prelaunch in the laboratory [31]. However, postlaunch evaluation shows that stripings in VIIRS $\mathrm{M}$ and I bands are not as significant as predicted. There was noticeable striping for the initial RSB images, but that was primarily due to the use of incorrect LUTs, and this issue was resolved after the LUT update in February 2012. All VIIRS bands including both RSB and TEB have minimal striping, which is in contrast to the significant striping in some MODIS bands. The magnitude of the residual striping in the $\mathrm{OC}$ bands is of the order of $\pm 5 \%$ of the typical OC radiance values, primarily in the blue bands (412 and $445 \mathrm{~nm}$ ), and the magnitude of the striping for these bands are comparable to that of the matching MODIS bands. The other OC bands have no noticeable striping.

For the TEB such as M12, it was found that striping can be as much as $\pm 5 \%$ of the typical radiance at high-scan angles. However, preliminary studies reveal that this type of striping at high-scan angles involving reflected solar radiation can be caused by small sensor azimuth-angle variations among the detectors. For example, the sensor azimuth-angle differences between detector 1 and 16 are of the order of 2 degrees near the end of the scan. When viewing non-Lambertian surfaces, the BRDF can introduce differences in the observed intensities by detector 1 or 16 . This in turn causes striping when observations from detector 16 are followed by those from detector 1 along the track direction. This phenomenon has been demonstrated in simulations through radiative transfer calculations [28] and presented at conferences (journal paper is in preparation). In general, the striping in VIIRS-TEB is significantly better 


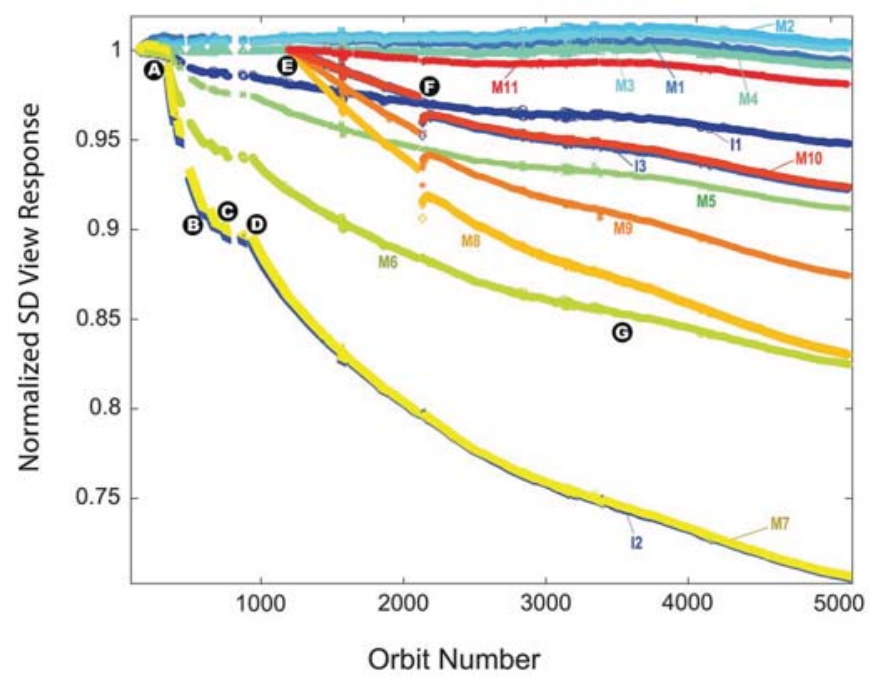

Fig. 7. VIIRS RSB band degradation seen from SD data.

than the corresponding MODIS bands. Overall, striping is mostly observed when viewing highly uniform scenes, such as the Antarctic snow and highly uniform ocean surfaces. The residual striping in the RSB will be further evaluatedin particular, on the effects of uneven polarization sensitivity across detectors [31].

The VIIRS DNB band has moderate striping at times when the instrument is illuminated by the sun and observing nighttime or terminator scenes, due to straylight issues for this band. Removing the DNB striping in the operational data processing can be complex due to the seasonal and geographical variations and therefore will require additional time to work through. Nevertheless, correction algorithms have been developed and offline analysis can be performed to reduce the striping on a case-by-case basis. As an example, the DNB striping in Fig. 1 was removed by simply applying threshold values through the image enhancement, although the ultimate solution will still rely on the radiometric correction algorithm for quantitative analysis.

3) VIIRS Optical Throughput Degradation and Response Stability: Shortly after the VIIRS nadir door was opened on November 21, 2011 (Fig. 7, Point A), it was found that the VIIRS-instrument responsivity degradation in the longer wavelength visible/near-infared RSB was much larger than expected, based on SD calibration. For example, by December 5, 2011 (Fig. 7, Point B), the M7 responsivity had already degraded $8 \%$ since the nadir door opened three weeks earlier. This is in sharp contrast to the Aqua MODISRSB responsivity degradation which is no more than $3.6 \%$ per year [23]. The VIIRS responsivity degradation has a spectral signature that is most prominent in band M7 and $\mathrm{I} 2$ at $0.86 \mu \mathrm{m}$ and is smaller in other bands such as M5, M6, M8-M10, and I1, I3. An extensive investigation was conducted led by the NASA anomaly resolution team. One of the hypotheses during the investigation was that the mirrors in the RTA may be degrading with a light exposure. Therefore, VIIRS telescope witness mirror (TWM) samples were tested in the laboratory, and spectral degradation with ultraviolet (UV) light exposure was found to be similar in degradation rate and spectral signature to what was seen in the VIIRS on-orbit responsivity decrease [32]. The TWM test also revealed that after 24 of UV exposure, mirror reflectance degraded $\sim 15 \%$ at $0.86 \mu \mathrm{m}$, and the degradation essentially stopped with 50 hours of UV exposure. However, since in addition to the RTA primary mirror, potentially up to three more RTA mirrors (although with decreasing impact) may be affected by the contamination, and the total combined degradation could be much larger than $15 \%$. This hypothesis led to several special configuration tests to the VIIRS instrument from December 9, 2011 to January 2, 2012 (Fig. 7, Point C to D), in which the telescope was stowed in antinadir position for three days, and then operated only during night, avoiding UV exposure to the telescope mirror. The degradation paused during the periods in which UV exposure was avoided, which pinpointed the immediate cause of the degradation as an UV-driven darkening of the RTA mirrors. It was ultimately learned that the root cause of this darkening is the contamination of the RTA mirrors with tungsten and tungsten oxide in the manufacturing process [32]-[34]. Subsequent on-orbit UV exposure leads to the mirror darkening in those spectral bands centered around $1 \mu \mathrm{m}$. It was predicted at that time that the SWIR bands (M8-M11) would also be affected, which was proven to be true after the cryocooler door was open and data from M8 to M11 became available after January 18, 2012 (Fig. 7, Point E).

The S-NPP spacecraft experienced an anomaly and went into a sun-pointing "safe mode" on March 24, 2012, after which the VIIRS began to recover by March 26, 2012. However, a step-jump shift of about $2 \%$ in linear gain or responsivity of M8-M11 was observed after the event (Fig. 7, Point F). The cause for this behavior is believed to be due to overheating of the cooler, which may have caused some permanent change. Nevertheless, the degradation resumed its trend after the event for all bands affected, although the degradation rate in general became slower. By summer 2012, M7 degradation rate dropped to about $0.7 \%$ per week from a few percent a week in the beginning of the mission. Finally, there appears to be a slight acceleration in the degradation after the summer of 2012 (around orbit 3500 in Fig. 7, Point G). However, this appears to have occurred for all bands including those not affected by the degradation earlier such as M1-M3. The cause for this change is believed to be error in the SD screen transmission LUT and this error will be corrected using an improved LUT derived from yaw maneuver data.

The VIIRS-SDR team has developed a strategy to mitigate the effects of the RTA mirror degradation through more frequent updates of the calibration scale factor LUTs to reduce the radiometric calibration bias and uncertainties. As of February 10, 2012, a weekly LUT update has been implemented, which significantly reduces the impact of degradation but with a residual effect of up to a $0.7 \%$ change between weekly updates. In August 2012, an operational code upgrade was implemented, which updates the RSB calibration scale factors on a scan-by-scan basis using predicted trends. This code change has reduced residual discontinuities in calibration to $0.1 \%$ or less (typically) between weekly LUT updates. However, there remains a time-varying calibration error of the 
order of $0.3 \%$ due to the uncertainties in predicting ahead the calibration scale factors and trends captured in the LUTs. The degradation is being closely monitored. Based on early studies, the current calibration-update scheme makes the degradation impact negligible for most EDR products, except for more calibration sensitive products such as the OC. A dynamic gain adjustment based on SD gain tracking on each orbit for the RSB is currently being investigated, and the algorithm will likely be implemented in 2013. This enhancement in processing will eliminate the discontinuities and time-varying predict-ahead calibration errors associated with the weekly LUT update process described above.

Another major impact of the degradation is the decreased SNR for those bands affected due to the reduced sensitivity. For example, the M7 low-gain SNR decreased from 631 in April, 2012, to 551 by September, 2012. Major efforts have been devoted to the prediction of this degradation and the SNR specification compliance over time. An earlier prediction by the instrument vendor estimated that the total degradation in the VIIRS-RTA optical throughput over the instrument life time (7 years or 450 equivalent UV exposure days) would be about $35 \%$ since launch. By then, the SNR would still have a positive margin of at least $6.5 \%$ for the M7 lowgain band and 35\% margin for the high-gain band [35]. As of October 23, 2012, the M7 responsivity has degraded about $30 \%$. However, the SNR is not linearly correlated with the responsivity, and the latest predicted SNR values have not changed significantly (Oudrari and Lei, 2012, personal communications). Other prediction models are also developed by the VIIRS-SDR team members, including both physicsbased and statistics-based models. While all models indicate that the degradation will continue at a decelerating rate, it is difficult to accurately predict the degradation over time.

Finally, since the mirror degradation is spectrally dependent, it is believed that the VIIRS band spectral response may be changing with the degradation for those bands affected. In other words, the ongoing degradation of VIIRS mirror reflectance is modulating the VIIRS-RSB-RSR. To study this effect, the TWM test data were used to simulate the impact after five years and at the end of mission life. Degradation modulated VIIRS-RSRs have been generated based upon an empirically tuned thin-layer physical degradation model developed by the NASA-VIIRS Characterization Support Team (VCST). It was predicted that up to $0.5 \%$ radiance error (with M1 being the largest) is expected for VIIRS-RSR after the four VIIRS mirrors are completely degraded at the end of mission life, assuming that the RSR used in producing the SDR data is not updated to reflect the spectral changes (otherwise, the impact would be minimal). A set of modulated RSRs has been produced for VIIRS by the VIIRS-SDR team, and made available to users for evaluation [9].

4) VIIRS SDR Intercomparison With Aqua/MODIS: It is essential to compare VIIRS-calibrated radiances with those of MODIS to ensure the product consistency. Any radiometric biases between them should be well understood and resolved if possible. Assessments immediately after the launch in the comparisons between VIIRS and MODIS show that many RSB had biases due to a number of issues, including the use of suboptimal LUTs, software issues, and instrument-throughput degradation, as discussed earlier. To ensure that the VIIRS measurements are consistent with those from other satellite radiometers such as MODIS, rigorous intercomparisons between VIIRS and Aqua MODIS have been performed at simultaneous nadir overpasses (SNOs) in the higher latitudes, as well as at lower latitudes [36]-[38].

The SNOs occur relatively frequently between S-NPP and Aqua satellites (every 2 to 3 days) due to the difference in orbital periods between these two satellites. For most satellite pairs, SNOs typically occur in the polar regions, and therefore most of the observations are over snow, which has unique spectral characteristics. As a result, the radiometric biases cannot be fully assessed for other land-cover types. Similarly, the geolocation differences cannot be assessed accurately due to the lack of high spatial frequency features in the polar regions, limiting the capability of the SNO method. Between S-NPP, Aqua, and NOAA-19, which are all afternoon satellites with different orbital periods, SNOs also occur in the low latitudes. The low-latitude SNO events occur when the highaltitude satellites such as S-NPP and NOAA-19 are flying above the low-altitude satellites such as Aqua for a large portion of the orbit (up to half an orbit) with their nadirs very close to each other (within $100 \mathrm{~km}$ ). The time differences are typically within $\sim 5$ min between Aqua and NOAA-19, and $\sim 10$ min between S-NPP and Aqua. This simultaneity is less stringent compared to the traditional SNO approach, which is of the order of $30 \mathrm{~s}$ [36], [37]. However, since the SNOs occur at low latitudes, it allows the intercomparison over a variety of land-cover types. The geolocation discrepancies can also be revealed using high spatial frequency features in earth scenes. While the SNO methodology is relatively mature [36], [37], its extension to the low latitudes is relatively new and is only available for satellite pairs in similar orbits (both S-NPP and Aqua are afternoon orbits). The SNOs are being routinely predicted using the latest orbital perturbation model (SGP4 v2008) and the results are readily available from the website at: https://cs.star.nesdis.noaa.gov/NCC/SNOPredictions. The daily S-NPP satellite trajectory can also be found at the same website.

Based on preliminary analysis with the SNO data, radiometric values for most channels nominally agree between VIIRS and MODIS. For the TEB, all VIIRS bands agree with similar bands of MODIS and AVHRR to within $0.2 \mathrm{~K}$, except VIIRS M12 versus MODIS B20 due to large RSR differences between them. During day time, the brightness temperature bias between VIIRS M12 and MODIS B20 over ocean is of the order of $0.7 \mathrm{~K}$, due to the RSR differences and reflected sunlight. At night time, the brightness temperature difference becomes minimal over ocean for these two bands. For the RSB, a bias of the order of 5\% was observed between VIIRS M1 and MODIS B8 (OC blue-band), which later was found due to viewing the angle-dependent biases (drifts) in MODIS Collection 5 data, and the bias has disappeared when compared with MODIS Collection 6. A bias of the order of $10 \%$ between VIIRS M5 and MODIS Band 1 is largely due to the RSR differences according to radiative transfer calculations such as with $6 \mathrm{~S}$ and MODTRAN. The biases in other matching 


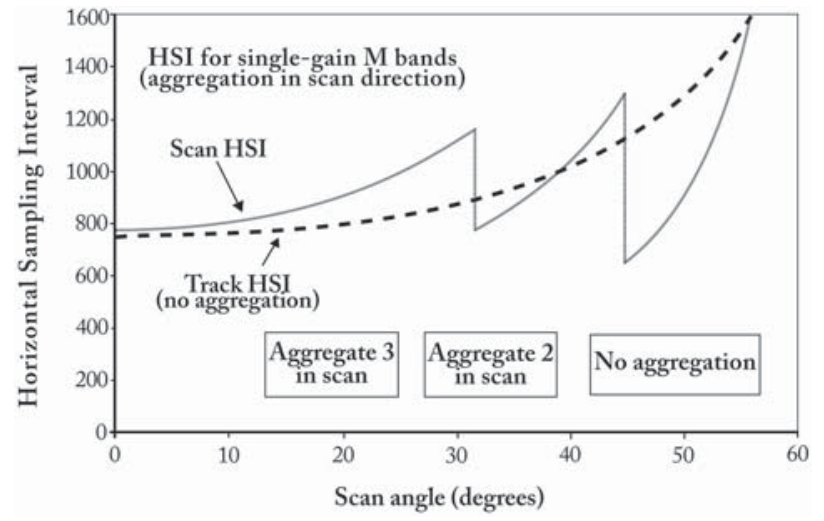

Fig. 8. Controlling VIIRS cross-track pixel growth in HSI with three aggregation zones.

bands are smaller but further investigation is needed to reduce uncertainties in the comparisons with a longer time series. The biases are monitored closely at SNO locations, as well as desert and the Antarctic Dome C sites.

Biases between VIIRS and AVHRR bands are larger, due to spectral response differences, as well as AVHRR traceability issues, with AVHRR Ch 1 being lower than both VIIRS- and MODIS-matching bands by approximately $9 \%$ [37]. The SNO analysis will be continuously used in the VIIRS, MODIS, and AVHRR analysis to develop time series for all matching bands between them, and the results will provide important feedback to the onboard calibration, which will lead to the establishment of consistent measurements among VIIRS, MODIS, and AVHRR. More extensive analysis of the biases between MODIS and VIIRS has been presented at conferences [38] and will be published in future articles.

It is noted that while the timing of the VIIRS nadir door opening was optimal for using the Antarctic Dome C calibration site for intercomparisons in the astral summer [39], unfortunately, the VIIRS LUTs used in data production were not finalized until early spring. As a result, analyses using the Dome C data have limited value in 2012, and therefore were primarily used for degradation monitoring using the band ratios. It is expected that the site can be used for more extensive analysis in 2013.

\section{VIIRS Geospatial Performance}

By design, VIIRS uses a unique approach of controlling cross-track scan spatial resolution through pixel aggregation, which limits the geometric pixel growth toward the end of the scan (Fig. 8). Although it is transparent to the users, the pixel aggregation is performed on the spacecraft for the single-gain bands, while on the ground for the dualgain bands. This is achieved in the across-track (along-scan) direction with a set of aggregation zones to average up to three raw measurement samples for each sample in the SDR. For example, the 3200 cross-track SDR samples for the M-bands are actually made by aggregating 6304 raw samples in three aggregation zones: 592 SDR samples are each aggregate of 3 raw samples in zone 1 near nadir, 368 aggregates of 2 in zone 2 , and the last 640 samples are not aggregated in zone 3 near end of the scan (Fig. 9). Pixel growth is a known problem



Fig. 9. VIIRS resolution, aggregation, and bow-tie deletion (Note: white areas overlap from one scan to the next, orange/purple areas denote bow-tie deletion, after Wolfe et al. 2012).

that has significantly affected the geometric performance of predecessor sensors such as MODIS and AVHRR (Fig. 10) [1]. As a result, the VIIRS spatial resolutions for the nadir and edge-of-scan data are more comparable (within a factor of two, in comparison to a factor of nearly five for MODIS). Compared to predecessor sensors, VIIRS also has a pixel shape as approximately square from nadir to end of the scan, while there are larger distortions in the pixel shape in previous sensors such as AVHRR, MODIS, and OLS (Fig. 10). VIIRS also employs "bow-tie removal" onboard to delete overlapped pixels (lines) along track at large scan angles, which removes redundant information and saves bandwidth for the data transmission (Fig. 9). This design feature does, however, introduce visual artifacts in the raw image due to the removal of mostly duplicated pixels on each side of a scan. These disappear when the image display is georeferenced and remapped into geographic projection. Finally, the VIIRS I bands are nested into M-bands $(2 \times 2$ I-band pixels to 1 M-band pixel scheme) through sampling alignment so that they have the same pixel coverage, meeting the band-to-band coregistration $(\mathrm{BBR})$ requirements discussed later.

For geolocation, knowledge of spacecraft ephemeris, attitude angles including roll, pitch, and yaw, alignment errors and instrument scan rate are used to geolocate the sensor data with high accuracy. In addition to providing separate ellipsoid geolocation fields (i.e., latitude, longitude, viewing, and solar angles) for the I-bands, M-bands, and DNB, terrain-corrected geolocation is also provided for the I-bands and M-bands. (Plans are underway to also provide the terraincorrected geolocation for the DNB.)

To verify the geolocation accuracy, the same methodology used for MODIS has been adopted for VIIRS. The controlpoint matching program developed at NASA, which has been used for MODIS for more than a decade, uses a library of over 1200 globally distributed ground control points chips of Landsat red band with $30-\mathrm{m}$ resolution clear subscenes. These chips are used to simulate images of VIIRS band I1 at 375-m nadir resolution with the scan geometry. The simulated images are then correlated with VIIRS images. Based on about four months of data since early 2012, the results show that the VIIRS geolocation accuracy has a track mean of 


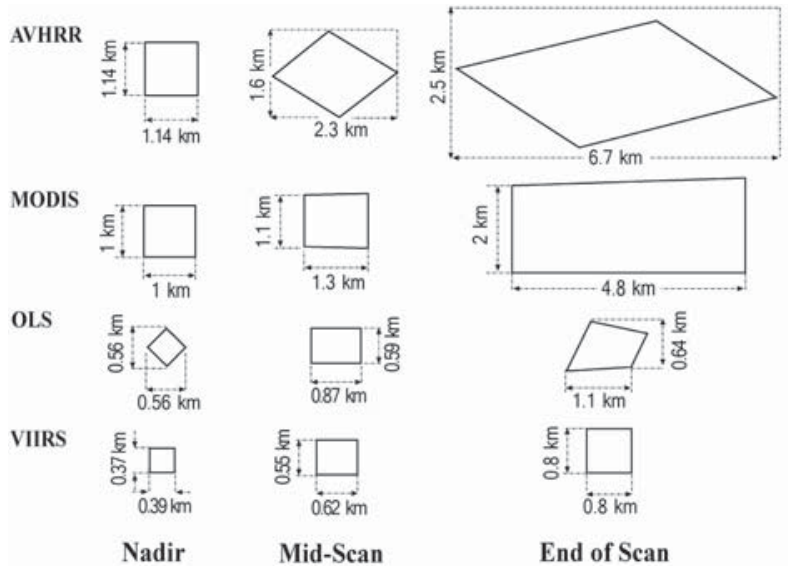

Fig. 10. VIIRS off-nadir footprint size growth in comparison with other instruments (not to scale).

$\sim 24 \mathrm{~m}$ and scan mean of $-13 \mathrm{~m}$, with root mean square error of 83 and $62 \mathrm{~m}$ in track and scan direction, respectively (all geolocation accuracy values are given in nadir equivalent units) [40]. In other words, the geolocation uncertainty is better than a quarter of an I-band pixel, which is excellent and comparable to that of MODIS. Other aspects of geospatial performance such as line spread function (LSF) and BBR have also been verified on-orbit with good results. For example, the BBRs for all bands are generally comparable to their prelaunch performance, meeting the better-than- $80 \%$ overlap requirement in both scan and track directions. Preliminary check of the LSF using such common targets as the Lake Pontchartrain Bridge near New Orleans and the moon shows that it is performing as expected, although additional study is required for more quantitative evaluation. Details can be found in [40] and future publications.

\section{Other InSTRUMENT ARTIFACTS, ISsues, AND Potential IMPACTS ON PRODUCTS}

Several other instrument artifacts and issues were identified in the VIIRS postlaunch calibration/validation process. Although their impacts on products are relatively small at this time, they should be closely monitored and adequately addressed. The following provides a summary.

1) VIIRS A-side versus B-side electronics calibration differences: It is known that the B-side was better characterized and analyzed prelaunch than that of the A-side, and therefore the B-side is preferred for routine operational use. However, it appears that the B-side electronics may have minor difficulties with the 1394 communications interface and spacecraft computer. The flight project is assessing whether the A-side may be more stable, and it is possible that VIIRS may be switched to the electronics A-side of the system to test this hypothesis. The VIIRS-SDR performance discussed here is based on the B-side calibration. If the electronics is switched to the A-side, the VIIRS performance will need to be reevaluated systematically.

2) M6-band radiance saturation and "fold-over": During the postlaunch cal/val, it was found that when the
M6 band saturates, any M6 radiance greater than Ls (Ls $=$ radiance at saturation) would lead to decreasing digital values with increased radiances. This gives the impression that these radiances are less than Ls. While the impact of this behavior should be small because M6 is used for atmospheric correction for the OC with low radiances, the saturated pixels needs to be flagged and identified in data processing so that users are advised to handle the data accordingly. For example, very high radiance values in M7 are usually indicative of fold over values in M6. As of October 23, 2012, the quality flags in the VIIRS-SDR data were modified to more accurately indicate the saturated and poor-quality radiance values. More detailed description about this issue can be found in internal reports (Moeller, personal communications, 2012).

3) Straylight contamination in the DNB: The DNB band performance is affected by straylight that contaminates the earth scene and introduces striping. The effect is dynamic and it depends on the terminator location at the spacecraft, which varies with season (Miller, personal communications, 2012). For example, during the northern hemisphere summer solstice, straylight has a moderate effect on the night-time DNB images in the Washington, DC region. A correction algorithm has been developed by the VIIRS-SDR team and will be implemented in the near future.

4) Reprocessing of early VIIRS-SDR data: It is recommended that VIIRS-SDR data before Feb. 10, 2012 should not be used for quantitative analysis due to issues with the LUT used in the ground-processing system. In general, reprocessing of the data before the implementation of the SD-based dynamic gain adjustments will be needed in the future if these data are to be used for the long-term time series analyses.

5) Instrument and spacecraft maneuvers and tests: Users should be aware that maneuvers and special tests are still being performed with VIIRS to better characterize the instrument performance. These include but are not limited to, the monthly lunar maneuver, and the quarterly blackbody warm-up-cool-down tests. During such events, the data may not be optimal. Data users are encouraged to contact the VIIRS-SDR team if any related issues arise.

Several other performance issues are being evaluated as part of the ongoing cal/val effort. It is known that a dual gain anomaly occurs approximately twice a day and affects two scan lines in each event. However, a software fix has been implemented in the ground processing with the MX6.3 and MX6.4 upgrade in the Interface Data Processing Segment as of October 2012. The VIIRS polarization sensitivity has been characterized prelaunch and shows uneven polarization sensitivity among the detectors in the RSB which might cause striping and will require additional studies. Similarly, focal plane scattering, optical crosstalk, and outof-band spectral response are found to be insignificant in the early assessment of the VIIRS performance but fur- 
ther quantitative studies are needed. A more thorough evaluation of the LUTs, such as those involving the use of the solar spectrum, is also needed to further reduce the uncertainties.

\section{VIIRS-SDR DATA DISTRIBUTION AND ACCESS}

The VIIRS data are divided into three levels [41]: the raw data records (RDRs or level 0) contain engineering and housekeeping data for spacecraft and sensor monitoring, and science data for SDR production; SDR or level 1 are calibrated radiance/reflectance and brightness temperatures with geolocation, and are the inputs to EDR algorithms for all applications; and EDRs or level 2 are application products such as those listed in Table I. All these data are available through the official data distribution and access systems.

For most data users and the general public, the Comprehensive Large-Array Stewardship System (CLASS) is the best option for accessing the VIIRS-SDR and EDR data. CLASS is an online data management system that provides users fast and easy access to environmental satellite data with their archived data products and documentation. The data-access latency depends on the status of the data with respect to such things as near-line storage. The CLASS web site for data access as well as related information can be found at: http://www.class.noaa.gov.

The data access from CLASS is open and free, although users will need to register an account online. A graphic user interface is provided for searching VIIRS SDR and EDR datasets. Once selected, a data order can be checked out. An email notification will be sent to the email address provided with instructions for downloading the data from the CLASS through ftp. If a large volume of data sets is needed from CLASS, automated scripts can be developed, or special arrangements can be made with the CLASS personnel. To facilitate the S-NPP data access, CLASS has recently set up a 100-TB ftp server, which is freely available at ftp://ftp-npp. class.ngdc.noaa.gov/ with a revolving 90 days of SDR and EDR data.

In addition to the CLASS, two centrals are recognized as the central hubs in the distribution and access of S-NPP/JPSS data. The NOAA/NESDIS facility at Suitland is one of the central hubs. The other central is the Air Force Weather Agency (AFWA) at Offutt Air Force Base, Omaha, NE. However, specific data access options are evolving. To support S-NPP launch, the Government Resource for Algorithm Verification, Independent Test, and Evaluation (GRAVITE) system is used for the distribution of RDR, SDR, and EDR to the team members. For local areas, direct broadcast is also available.

It should be noted that the VIIRS data volume is relatively large. One quartet granule $(\sim 5.69 \mathrm{~min})$ of VIIRS data (including geolocation) takes approximately 3.6 GB of storage, including $\sim 1.23 \mathrm{~GB}$ for the M-bands, $1.8 \mathrm{~GB}$ for the I-bands, and $0.6 \mathrm{~GB}$ for the DNB band. The VIIRSSDR data are stored in hierarchical data format 5 (HDF5), which can be easily processed with standard HDF5 tools such as IDL, ENVI, MATLAB, and HDFViewer. Additional information about VIIRS-SDR including the VIIRS-SDR user's guide [41] as well as sample software can be found at https://cs.star.nesdis.noaa.gov/NCC/VIIRS.

\section{CONCLUSION}

The launch of the S-NPP with the key instrument VIIRS ushers in a new generation of capabilities for operational environmental observations following the legacy AVHRR and MODIS. Through the postlaunch calibration/validation, it was found that the VIIRS instrument is performing very well, and producing high-quality data for a variety of applications. Preliminary results show that the VIIRS has very low noise and high signal-to-noise ratio, exceeding the performance specifications. The calibration impact due to the greater-thanexpected optical throughput degradation is being mitigated by more frequent calibration updates and an upgrade to the operational calibration code. The evolving degradation and related impacts are being closely monitored by the VIIRS-SDR team. Intercomparisons with MODIS observations at simultaneous nadir overpasses show consistency between VIIRS and MODIS observations, and the small biases, as well as several other issues are being further investigated to meet the needs of future applications. The VIIRS-SDR team is working diligently to address issues found in the postlaunch calibration/validation and is providing critical support to the S-NPP and JPSS mission.

\section{ACKNOWLEDGMENT}

The authors would like to thank the VIIRS-SDR team members from NOAA, The Aerospace Corporation, NASA, University of Wisconsin, MIT Lincoln Laboratory, Raytheon, and Northrop Grumman for their dedicated support in the prelaunch and postlaunch calibration/validation of VIIRS SDR data. Thanks are extended to the NASA mission operations team, anomaly resolutions team, NOAA Data Processing and Algorithms segment, and VIIRS-EDR team members for collaboration in evaluating the product quality. The manuscript contents are solely the opinions of the authors and do not constitute a statement of policy, decision, or position on behalf of NOAA or the U.S. government.

\section{REFERENCES}

[1] T. Lee, S. Miller, C. Schueler, S. Miller, "NASA MODIS previews NPOESS VIIRS capabilities," Weather Forecast., vol. 21, no. 4, pp. 649-655, 2006.

[2] R. P. Murphy, P. E. Ardanuy, F. Deluccia, J. E. Clement, and C. Schueler "The visible infrared imaging radiometer suite," in Earth Science Satellite Remote Sensing, vol. 1, New York, USA: Springer-Verlag, 2006, pp. 199-223.

[3] K. D. Hutchinson and A. P. Cracknell, Visible Infrared Imager Raidometer Suite-A New Operational Cloud Imager. New York, USA: Taylor \& Francis, 2006, p. 230.

[4] T. E. Lee, S. D. Miller, F. J. Turk, C. Schueler, R. Julian, S. Deyo, P. Dills, and S. Wang, "The NPOESS VIIRS day/night visible sensor," Bull. Amer. Meteorol. Soc., vol. 87, pp. 191-199, Feb. 2006.

[5] C. J. Tucker and K. A. Yager, "Ten years of MODIS in space: Lessons learned and future perspectives," Eur. J. Remote Sens., vol. 43, no. 3, pp. 7-18, 2011.

[6] M. Roman, I. Csiszar, C. Justice, J. Key, J. Privette, S. Devadiga, C. Davisdon, R. Wolfe, and E. Masuoka, "Status of the suomi NPP visible/infrared imager radiometer suite's (VIIRS) land environmental data records (EDRs) after early evaluation of on-orbit performance," in Proc. IEEE Int. Geosci. Remote Sens. Symp., Munich, Germany, 2012, pp. 1084-1087. 
[7] C. Justice, E. Vermote, J. Privette, and A. Sei, "The evolution of U.S. moderate resolution optical land remote sensing from AVHRR to VIIRS," in Land Remote Sensing and Global Environmental Change (Remote Sensing and Digital Image Processing), vol. 11. New York, USA: Springer-Verlag, 2011, pp. 781-806.

[8] A. Ignatov, P. Minnett, R. Evans, D. May, R. Arnone, J. Stroup, J. Sapper, S. Jackson, P. LeBorgne, X. Liang, B. Peternko, P. Dash, Y. Kihai, M. Bouali, F. Xu, and K. Saha, "SST from NPP/VIIRS," in Proc. IEEE Int. Geosci. Remote Sens. Symp., Munich, Germany, 2012, no. 1440 .

[9] W. P. Menzel, B. A. Baum, K. L. Strabala, and R. A. Frey, "Cloud top properties and cloud phase-Algorithm theoretical basis document," Goddard Space Flight Center, Greenbelt, MD, USA, Tech. Rep. ATBDMOD-04, 2002.

[10] R. L. Vogel, J. L. Privette, and Y. Yu, "Creating proxy VIIRS data from MODIS: Spectral transformations for mid- and thermal-infrared bands," IEEE Trans. Geosci. Remote Sens., vol. 46, no. 11, pp. 3768-3782, Nov. 2008.

[11] S. D. Miller and R. E. Turner, "A dynamic lunar spectral irradiance data set for NPOESS/VIIRS day/night band nighttime environmental applications," IEEE Trans. Geosci. Remote Sens., vol. 47, no. 7, pp. 2316-2329, Jul. 2009.

[12] R. M. Durham, "Sensor specification for the visible infrared imager radiometer suite," Raytheon Systems Company, Goleta, CA, USA, Tech. Rep. PRT PS 154640-101A, 2002.

[13] Joint Polar Satellite System (JPSS) VIIRS Performance Requirements Document Goddard Space Flight Center, Greenbelt, MD, USA, 2012.

[14] C. Moeller, J. McIntire, T. Schwarting, D. I. Moyer, and J. Costa, "Suomi NPP VIIRS spectral characterization: Understanding multiple releases," Proc. SPIE, vol. 8510, p. 85101S Oct. 2012

[15] W. Chen and R. Lucke, "Out-of-band correction for multispectral remote sensing," IEEE Trans. Geosci. Remote Sens., vol. PP, no. 99, pp. 1-8, Aug. 2012.

[16] F. Deluccia and C. Cao, "VIIRS SDR calibration/validation operations concept (OPSCON) document," unpublished.

[17] C. Cao, X. Xiong, and F. Weng, "NPP VIIRS SDR postlaunch calibration/validation: An overview of progress, challenges, and the way forward," Proc. SPIE, vol. 8510, p. 851014, Oct. 2012.

[18] F. Deluccia, C. Cao, X. Xiong, R. Wolfe, H. Oudrari, K. Chiang, E. Johnson, S. Mills, L. Liao, D. Moyer, and K. Rausch, "Suomi NPP VIIRS sensor data record quality," in Proc. IEEE Trans. Geosci. Remote Sens., Munich, Germany, 2012, no. 1597.

[19] X. Xiong, K. Chiang, J. McIntire, H. Oudrari, A. Wu, M. Schwaller, and J. Butler, "Early assessment of VIIRS on-orbit calibration and support activities," in Proc. IEEE Int. Geosci. Remote Sens. Symp., Munich, Germany, Jul. 2012, pp. 7189-7192.

[20] F. Deluccia and C. Cao, "NPP VIIRS instrument characteristics, measurements and sensor data record production," in Proc. Amer. Meteorol. Soc. Annu. Conf., 2012.

[21] B. Guenther, X. Xiong, V. V. Salomonson, W. L. Barnes, and J. Young, "On-orbit performance of the earth observing system moderate resolution imaging spectroradiometer; first year of data," Remote Sens. Environ., vol. 83, pp. 16-30, Feb. 2002.

[22] X. Xiong, B. N. Wenny, A. Wu, W. Barnes, and V. Salomonson, "Aqua MODIS thermal emissive bands on-orbit calibration, characterization, and performance," IEEE Trans. Geosci. Remote Sens., vol. 47, no. 3, pp. 803-814, Mar. 2009.

[23] X. Xiong, J. Sun, X. Xie, W. Barnes, and V. Salomonson, "On-orbit calibration and performance of aqua MODIS reflective solar bands," IEEE Trans. Geosci. Remote Sens., vol. 48, no. 1, pp. 535-546, Jan. 2010.

[24] A. H. Johnston, "Radiation damage of electronic and optoelectronic devices in space," in Proc. 4th Int. Workshop Radiat. Effects Semicond. Devices Space Appl., Oct. 2000.

[25] J. C. Cardema, K. W. Rausch, N. Lei, D. I. Moyer, and F. Deluccia, "Operational calibration of VIIRS reflective solar band sensor data records," Proc. SPIE, vol. 8510, p. 851019, Oct. 2012.

[26] H. Oudrari, F. Deluccia, J. McIntire, D. I. Moyer, K. Chaing, X. Xiong, and J. J. Butler, "Preliminary assessment of suomi-NPP VIIRS on-orbit radiometric performance," Proc. SPIE, vol. 8510, p. 851011, Oct. 2012.

[27] J. Butler, X. Xiong, R. A. Barnes, F. S. Patt, J. Sun, and K. Chiang, "An overview of NPP VIIRS calibration maneuvers," Proc. SPIE, vol. 8510, p. 85101 J, Oct. 2012 .

[28] Q. Liu, C. Cao, and F. Weng, "NPP VIIRS emissive band radiance calibration and analysis," Proc. SPIE, vol. 8510, p. 85101E, Oct. 2012.

[29] D. Moyer, J. McIntire, F. Deluccia, B. Efremova, K. Chaing, and X. Xiong, "VIIRS thermal emissive bands calibration algorithm and on-orbit performance," Proc. SPIE, vol. 8510, p. 85101D, Oct. 2012.
[30] E. Johnson and C. Ranshaw, "VIIRS emissive band radiometric performance trending," Proc. SPIE, vol. 8510, p. 85101F, Oct. 2012.

[31] B. Guenther, F. Deluccia, B. Gao, P. Zhai, and R. Lucke, "An introductory look at handling polarization characterization in the level 1 calibration product in the NPP VIIRS sensor," in Proc. IEEE Int. Geosci. Remote Sens. Symp., Vancouver, CA, USA, Jul. 2011.

[32] F. Deluccia, D. I. Moyer, E. H. Johnson, K. W. Rausch, N. Lei, K. Chiang, and X. Xiong, "Discovery and characterization of on-orbit degradation of the VIIRS rotating telescope assembly," Proc. SPIE, vol. 8510 , p. $85101 \mathrm{~A}$, Oct. 2012

[33] G. Iona, E. H. Johnson, M. Ayers, F. Deluccia, L. Graziani, B. Guenther, B. Kennedy, C. J. Kent, R. Lambeck, X. Xiong, and E. Waluschka, "VIIRS on-orbit optical anomaly: Lessons learned," Proc. SPIE, vol. 8510, p. 85101C, Oct. 2012.

[34] Y. Mo, R. O. Dillon, and P. G. Snyder, "Visible and infrared photochromic properties of amorphous $\mathrm{WO}_{3-\mathrm{x}}$ films," J. Vac. Sci. Technol. A, Vac., Surf., Films, vol. 17, no. 5, pp. 2933-379 2938, Sep. 1999.

[35] V. Murgai, N. R. Nelson, E. H. Johnson, and K. E. Yokoyama, "VIIRS VisNIR/SMWIR end-of-life sensitivity predictions," Proc. SPIE, vol. 8510, p. 85101L, Oct. 2012.

[36] C. Cao, M. Weinreb, and X. Hui, "Predicting simultaneous nadir overpasses among polar-orbiting meteorological satellites for the intersatellite calibration of radiometers," J. Atmospheric Ocean. Technol., vol. 21, pp. 537-542, Apr. 2004.

[37] C. Cao, X. Xiong, A. Wu, and X. Wu, "Assessing the consistency of AVHRR and MODIS L1B reflectance for generating fundamental climate data records," J. Geophys. Res., vol. 113, p. D09114, May 2008.

[38] C. Cao, S. Uprety, S. Blonski, and Q. Liu, "Establishing radiometric consistency among VIIRS, MODIS, and AVHRR using SNO and SNOx methods," in Proc. IEEE Int. Geosci. Remote Sens. Symp., Munich, Germany, Jul. 2012, pp. 6928-6931.

[39] C. Cao, S. Uprety, X. Xiong, J. Xiong, A. Wu, P. Jing, D. Smith, G. Chander, N. Fox, and S. Ungar, "Establishing the antarctic dome C community reference standard site toward consistent measurements from Earth observation satellites," Can. J. Remote Sens., vol. 36, no. 5, pp. 498-513, 2010.

[40] R. Wolfe, G. Lin, N. Masahiro, K. P. Tewari, and E. L. Montano, "NPP VIIRS early on-orbit geometric performance," Proc. SPIE, vol. 8510, p. 851013 , Oct. 2012.

[41] C. Cao, X. Xiong, F. Deluccia, Q. Liu, S. Blonski, D. Pogorzala, and H. Oudrari. "VIIRS SDR user's guide," U.S. Dept. Commerce, NOAA, Silver Spring, MD, USA, Tech. Rep. NESDIS 142, Feb. 2013.

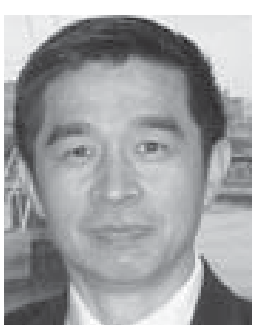

Changyong Cao received the B.S. degree in geography from Peking University, Beijing, China, 1982, graduate training from the Chinese Academy of Sciences, Beijing, China, and the Ph.D. degree in geography specializing in remote sensing from Louisiana State University, Baton Rouge, LA, USA, in 1992

$\mathrm{He}$ is a Research Physical Scientist with the NOAA/NESDIS/Center for Satellite Applications and Research. He specializes in the calibration of radiometers onboard NOAA's Operational Environmental Satellites, and is the managerial lead for the S-NPP VIIRS SDR team. He has made significant contributions to the international and inter-agency satellite community, including the Committee on Earth Observation Satellites and World Meteorological Organization Global Space-based Inter-Calibration System. Before joining the NOAA in 1999, he was a Senior Scientist with five years of aerospace industry experience supporting NASA projects, and three years of university teaching experience.

Dr. Cao was the recipient of two gold and one silver medal honored by the U.S. Department of Commerce for his scientific and professional achievements. 


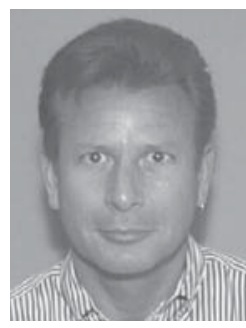

Frank J. De Luccia received the B.S. degree in physics and mathematics from the Massachusetts Institute of Technology, Cambridge, MA, USA, in 1974, and the M.A. and Ph.D. degrees in physics from Harvard University, Cambridge, MA, in 1976 and 1979 , respectively.

$\mathrm{He}$ is Senior Project Engineer with the Joint Operational Programs, The Aerospace Corporation, El Segundo, CA, USA. He has worked in satellite remote sensing supporting the DMSP, NPOESS, and JPSS programs since 1994. He joined the Aerospace Corporation in 1984 and has supported a variety of government programs, specializing in systems engineering, requirements analysis and flowdown, sensor system conceptual design, and sensor performance analysis. From 1980 to 1984, he was with Rockwell International (now Boeing), Anaheim, CA where he modeled, designed, and tested infrared detectors and readouts. From 1979 to 1980, he was a Visiting Member with the Institute for Advanced Study, Princeton, NJ, USA, engaged in research in theoretical particle physics.

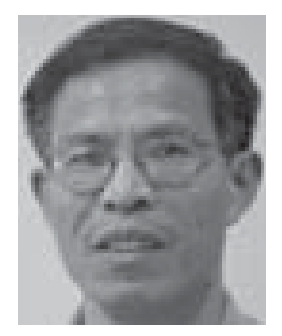

Xiaoxiong Xiong (M'12) received the B.S. degree in optical engineering from the Beijing Institute of Technology, Beijing, China, and the Ph.D. degree in physics from the University of Maryland, College Park, MD, USA.

$\mathrm{He}$ is an Optical Physicist with NASA Goddard Space Flight Center (GSFC), Greenbelt, MD, USA. $\mathrm{He}$ is currently serving as the MODIS Project Scientist and the technical lead for both the MODIS Characterization Support Team (MCST) and the VIIRS Calibration Support Team. Before joining the NASA/GSFC, he had also worked in optical instrumentation, nonlinear optics, laser and atomic spectroscopy, and resonance ionization mass spectrometry at universities, industry, and the National Institute of Standards and Technology.

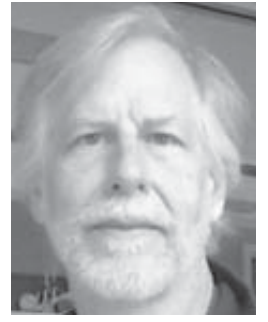

Robert Wolfe received the B.S. degree in math and physics from Bridgewater College, Bridgewater, VA, USA, in 1980.

$\mathrm{He}$ has worked in satellite remote sensing and computer science for 32 years. Starting early in the mission, he contributed to NASA's Earth Observating System (EOS) Moderate Resolution Imaging Spectroradiometer (MODIS) science support team in areas including integration and testing of MODIS science algorithms and data processing. Over the last seven years, he has led NASA Science Data Segment's (SDS's) effort to integrate and test operational Land algorithms for the Visible Infrared Imager Radiometer Suite system that is flying on the Suomi National Polar-orbiting Partnership (SNPP) satellite. He has led the team that built and operates the SNPP SDS Land Product Evaluation and Analysis Element. He has also led the development of advanced algorithms and innovative techniques to Earth-locate (geolocate) and grid land science data.

He is a member of the NPP Science Team (for geolocation and validation) and the EOS Terra Deputy Project Scientist. His current research interests include using satellite remote sensing to characterize global impervious surface area and heat signatures of urban settlements. He has authored over 80 scientific, technical, and symposia papers. He is a member of the IEEE Geoscience and Remote Sensing Society and the American Geophysical Union.

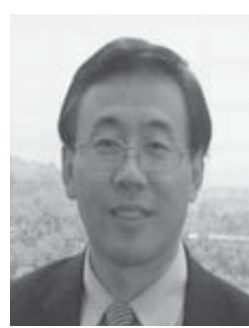

Fuzhong Weng received the Ph.D. degree from Department of Atmospheric Science, Colorado State University, Fort Collins, CO, USA, in 1992.

He is the Acting Chief of the Satellite Meteorology and Climatology Division, NOAA/NESDIS/Center for Satellite Applications and Research. He joined NOAA as a Research Scientist in 1992 and has been at NOAA since then on microwave remote sensing, instrument calibration, radiative transfer modeling and satellite data assimilation. From 2002 to 2005, he served as the Deputy Director of the U.S. Joint Center for Satellite Data Assimilation, Boulder, CO. From 2005 to 2009, he was the Chief of Sensor Physics Branch, NOAA/NESDIS.

Dr. Weng was selected by NOAA/NASA as the U.S. Joint Polar Satellite System Sensor Science Chair in 2010. He was the first recipient of the 2000 NOAA David Johnson Award for his outstanding contributions to satellite microwave remote sensing fields and the utilization of satellite data in the NWP models. He was the recipient of the U.S. Department of Commerce Gold Medal Award in 2005 for his achievement in satellite data assimilation and the NOAA bronze medal for leading successful NOAA18 instrument calibration, and the 2009 NOAA Administrator Award for his developments of powerful radiative transfer model for satellite data assimilation. 